\title{
Qualitatively adequate numerical modelling of spatial SIRS-type disease propagation
}

\author{
István Faragó ${ }^{1,3}$ and Róbert Horváth ${ }^{\otimes 2,3}$ \\ ${ }^{1}$ Eötvös Loránd University, 1/C Pázmány Péter sétány, Budapest, H-1117, Hungary \\ ${ }^{2}$ Budapest University of Technology and Economics, 1 Egry J. utca, Budapest, H-1111, Hungary \\ ${ }^{3}$ MTA-ELTE NumNet Research Group, 1/C Pázmány Péter sétány, Budapest, H-1117, Hungary
}

Appeared 11 August 2016

Communicated by Tibor Krisztin

\begin{abstract}
The aim of this paper is the investigation of some discrete iterative models that can be used for modeling spatial disease propagation. In our model, we take into account the spatial inhomogenity of the densities of the susceptible, infected and recovered subpopulations and we also suppose vital dynamics. We formulate some characteristic qualitative properties of the model such as nonnegativity and monotonicity and give sufficient conditions that guarantee these properties a priori. Our discrete model can be considered as some discrete approximation of continuous models of the disease propagation given in the form of systems of partial or integro-differential equations. In this way we will be able to give conditions for the mesh size and the time step of the discretisation method in order to guarantee the qualitative properties. Some of the results are demonstrated on numerical tests.
\end{abstract}

Keywords: differential equations, epidemic models, qualitative properties of systems of PDEs, nonnegativity, finite difference method.

2010 Mathematics Subject Classification: 34C60, 35B09, 65M06.

\section{Introduction}

The construction of mathematical models is one of the tools of the understanding of the mechanism of communicable diseases. These models can predict how to prevent the outbreak of an epidemic or can give ideas how to curb an epidemic with appropriate and affordable means (e.g. hygiene, vaccination).

The most popular and well investigated models are the so-called compartmental models $[1,3,4,7,10]$. In these models the population is considered to be homogeneously mixed and the individuals are classified according to their relation to the disease. For example, the so-called SIRS-model [4] has the form

$$
\begin{aligned}
S^{\prime} & =-a S I+d I+(c+d) R, \\
I^{\prime} & =a S I-(b+d) I, \\
R^{\prime} & =b I-(c+d) R,
\end{aligned}
$$

\footnotetext{
${ }^{\bowtie}$ Corresponding author. Email: rhorvath@math.bme.hu
} 
where $S=S(t), I=I(t)$ and $R=R(t)$ denote, respectively, the number of susceptible, infective and recovered individuals as a function of time $t$. The contact rate $a$ and recovery coefficient $b(1 / b$ is the infectious period) are positive known numbers. The mortality rate $d(1 / d(d \neq 0)$ is the expected life time) is a nonnegative number. The total population is maintained by the compensation of the mortality with an equal birth input $d(S+I+R)$ in the susceptible class. The recovered individuals become susceptible again with the rate $c$. The special condition $c=d=0$ (so-called SIR-model) means that there is no vital dynamics in the model (birth and death are neglected) and the recovered individuals do not become susceptible again.

The compartmental model cannot take into account the spatial distribution of the individuals. In order to bring also the spatial dependence into the picture we can divide the original population into subpopulations (metapopulations) according to some geopolitical considerations and connect them somehow into a network by some prescribed rules for the disease propagation [3]. Another possibility is that we take into account the movement of the individuals. This results in a partial differential equation of reaction-diffusion type [4,7-9]. In order to obtain a partial differential equation with spatial dependence but without the movement of the individuals, Kendall [9] suggested changing the term $a I$ in (1.1) to the integral

$$
\int_{N(x)} W\left(\left\|x^{\prime}-x\right\|\right) I\left(x^{\prime}, t\right) \mathrm{d} x^{\prime}
$$

This integral describes how the infectives at the points $x^{\prime}$ affect the infection of the susceptibles at the point $x$. The nonnegative weighting function $W$ is supposed to depend only on the distance of the points $x^{\prime}$ and $x$, and $N(x)$ denotes a prescribed neighbourhood of the point $x$. With the above modification we arrive at the system of integro-differential equations

$$
\begin{aligned}
S_{t}^{\prime}(x, t) & =-\left(\int_{N(x)} W\left(\left\|x^{\prime}-x\right\|\right) I\left(x^{\prime}, t\right) \mathrm{d} x^{\prime}\right) S(x, t)+d I(x, t)+(c+d) R(x, t), \\
I_{t}^{\prime}(x, t) & =\left(\int_{N(x)} W\left(\left\|x^{\prime}-x\right\|\right) I\left(x^{\prime}, t\right) \mathrm{d} x^{\prime}\right) S(x, t)-(b+d) I(x, t), \\
R_{t}^{\prime}(x, t) & =b I(x, t)-(c+d) R(x, t)
\end{aligned}
$$

(equipped with some initial conditions), where now $S=S(x, t), I=I(x, t)$ and $R=R(x, t)$ depend also on the spatial position and give the densities of the corresponding compartments of the population.

For ordinary differential equation models the number and the type of the equilibria are generally investigated. while for partial differential equation models travelling waves or nontrivial epidemic states are sought. However, only a little attention is devoted to obtaining qualitatively adequate solutions of different continuous and discrete models (e.g. $[5,11])$. In this paper, we will investigate some consistent discretizations of the system (1.3) from the qualitative point of view. We will suppose that the spatial dimension of the problem is one, and that $N(x)$ is a symmetric interval around any fixed point $x$.

The paper is organized as follows. In Section 2, we define a discrete space-time disease propagation model. This model will include also vital dynamics, and so it can be considered as a generalization of the results of paper [6]. We give sufficient conditions that guarantee some basic qualitative properties of the models. Because the models can be considered as certain discretizations of the system (1.3), we will be able to give a priori conditions for the spatial mesh size and the time step in Section 3. We close the paper with some numerical test examples in Section 4. 


\section{A discrete space-time disease propagation model}

In this section we construct a discrete one-step iteration model of spatial disease propagation and give the conditions of its qualitative properties.

We suppose that the population is located in one-dimension along the segment $L=[0, l]$ $(l>0)$ and that the individuals do not have spatial movements. The last property essentially means that the speed of the individuals can be neglected compared to the propagation speed of the disease.

The interval $L$ is divided into $0<N \in \mathbb{N}$ congruent subintervals with length $h=l / N$ : $L_{k}=[(k-1) h, k h](k=1, \ldots, N)$. We set a time step $\tau>0$. The density of the susceptible individuals is denoted by $s_{k}^{n}$ on $L_{k}$ and at the time instant $n \tau$. Thus $h s_{k}^{n}$ means the number of the susceptibles. Similar notation is used for the infected and recovered individuals: $i_{k}^{n}$ and $r_{k}^{n}$. Furthermore, we introduce the vectors $s^{n}=\left[s_{1}^{n}, \ldots, s_{N}^{n}\right]^{T} \in \mathbb{R}^{N}, i^{n}=\left[i_{1}^{n}, \ldots, i_{N}^{n}\right]^{T} \in \mathbb{R}^{N}$ and $r^{n}=\left[r_{1}^{n}, \ldots, r_{N}^{n}\right]^{T} \in \mathbb{R}^{N}$, where the superscript $T$ denotes the matrix transpose. In order to simplify the notations, in vector operations we will apply a general convention like it is defined in Matlab: if an operation cannot be carried out in a standard way, then it must be computed elementwise. The relations are also meant elementwise.

We will consider the following discrete one-step iteration model for the disease propagation

$$
\begin{aligned}
\frac{s^{n+1}-s^{n}}{\tau}= & -\left(\sigma s^{n+1}+(1-\sigma) s^{n}\right) p^{n}+d\left(\sigma i^{n+1}+(1-\sigma) i^{n}\right) \\
& +(c+d)\left(\sigma r^{n+1}+(1-\sigma) r^{n}\right), \\
\frac{i^{n+1}-i^{n}}{\tau}= & \left(\sigma s^{n+1}+(1-\sigma) s^{n}\right) p^{n}-(b+d)\left(\sigma i^{n+1}+(1-\sigma) i^{n}\right), \\
\frac{r^{n+1}-r^{n}}{\tau}= & b\left(\sigma i^{n+1}+(1-\sigma) i^{n}\right)-(c+d)\left(\sigma r^{n+1}+(1-\sigma) r^{n}\right) .
\end{aligned}
$$

Here $n=0,1, \ldots$ and $s^{0}, i^{0}$ and $r^{0}$ are given initial vectors. The parameters $b>0$ and $c, d \geq 0$ are given real numbers. (The coincidence with the parameters in (1.1) is intentional because the discrete model will be considered later as a certain discretization of some modification of the continuous model.) The parameter $\sigma$ is a fixed weighting parameter from the interval $[0,1]$. As it is usual, if $\sigma=0$ then the iteration process is called explicit otherwise it is implicit. The vector $p^{n} \in \mathbb{R}^{N}$ is derived from the vector $i^{n}$ and the mesh size $h$, and it describes the dynamics of the process how the susceptible individuals become infected.

It is a natural requirement for the models of any real life phenomenon that the solutions of the continuous/discrete models must possess some basic adequate qualitative properties of the original process. In the present case for the discrete model (2.1) such qualitative properties are as follows. Suppose $s^{0}, i^{0}$ and $r^{0}$ are nonnegative vectors.

[P1] Because the individuals do not move, the total size of the population at a given spatial position cannot change in time. This means that $s^{n}+i^{n}+r^{n}$ must be constant, that is independent of $n$.

[P2] The number of the susceptible, infective and recovered members must be nonnegative. That is $s^{n}, i^{n}$ and $r^{n}$ must be nonnegative if $s^{0}, i^{0}$ and $r^{0}$ are nonnegative.

[P3] If $c=d=0$, then besides property [P2] we also require that the number of the susceptibles cannot grow and the number of the recovered cannot decrease in time. That is $s^{n}$ is a nonincreasing and $r^{n}$ is a nondecreasing function of $n$ (elementwise). 
The validity of the qualitative properties [P1]-[P3] can be guaranteed by the following lemmas and theorems.

Lemma 2.1. Let $n \in \mathbb{N}$ be any fixed natural number. Then

$$
s^{n}+i^{n}+r^{n}=s^{n+1}+i^{n+1}+r^{n+1},
$$

that is property [P1] is satisfied without any condition.

Proof. The statement simply follows by adding the three equations in (2.1).

The maximum element of the vector $s^{0}+i^{0}+r^{0}$ will be denoted by $M$. Based on the previous lemma, hence $M=\max \left(s^{n}+i^{n}+r^{n}\right)$ for all $n$ is also true.

Lemma 2.2. If conditions

$$
\begin{aligned}
& \text { (C1) } \tau(\max \{c, b\}+d)(1-\sigma) \leq 1, \\
& \text { (C2) } p^{n} \geq 0 \text { and } \tau(1-\sigma) p^{n} \leq 1
\end{aligned}
$$

are satisfied then $s^{n} \geq 0, i^{n} \geq 0, r^{n} \geq 0$ imply that $s^{n+1} \geq 0, i^{n+1} \geq 0, r^{n+1} \geq 0$. When $c=d=0$ then the monotonicity conditions $s^{n+1} \leq s^{n}$ and $r^{n+1} \geq r^{n}$ are also true.

Proof. We reorder the equations in (2.1) such that we put the vectors with the superscript $n+1$ into the left-hand sides and the other terms to the right-hand sides. We obtain the system of linear equations

$$
A_{n}\left[\begin{array}{l}
s^{n+1} \\
i^{n+1} \\
r^{n+1}
\end{array}\right]=B_{n}\left[\begin{array}{c}
s^{n} \\
i^{n} \\
r^{n}
\end{array}\right]
$$

for the unknown vectors $s^{n+1}, i^{n+1}, r^{n+1}$, where

$$
\begin{aligned}
& A_{n}= {\left[\begin{array}{ccc}
1+\tau \sigma p^{n} & -\tau d \sigma & -\tau(c+d) \sigma \\
-\tau \sigma p^{n} & 1+\tau(b+d) \sigma & 0 \\
0 & -\tau b \sigma & 1+\tau(c+d) \sigma
\end{array}\right] } \\
& B_{n}=\left[\begin{array}{ccc}
1-\tau(1-\sigma) p^{n} & \tau d(1-\sigma) & \tau(c+d)(1-\sigma) \\
\tau(1-\sigma) p^{n} & 1-\tau(b+d)(1-\sigma) & 0 \\
0 & \tau b(1-\sigma) & (1-\tau(c+d)(1-\sigma))
\end{array}\right] .
\end{aligned}
$$

We recall that here the matrix-vector product is meant in the usual way and vectors are multiplied by vectors elementwise. Moreover, in the matrix $B_{n}$, the vector $p^{n}$ generally depends on the vector $i^{n}$.

It follows directly from conditions $(\mathrm{C} 1)-(\mathrm{C} 2)$ that $B_{n}$ is nonnegative. Hence the nonnegativity of the vectors $s^{n}, i^{n}$ and $r^{n}$ yields that the right-hand side of the system is nonnegative.

It is known that if a square matrix $A$ has nonpositive offdiagonal elements and there is a positive vector $g>0$ such that $A g>0$ then $A$ is nonsingular and its inverse is nonnegative. In this case $A$ is called an M-matrix (e.g. [2]).

Under the conditions (C1)-(C2) the transpose of $A_{n}$ is an M-matrix. The offdiagonal elements are trivially nonpositive. Moreover, the product with the positive vector $g=[1,1,1]^{T}$ is $[1,1,1]^{T}$, which is a positive vector. This implies that the transpose matrix is nonsingular and its inverse is nonnegative. Thus, the original matrix is also nonsingular and its inverse is nonnegative. We note that it follows from the above facts that the original matrix is also an M-matrix. 
Because the right-hand side vector in (2.3) is nonnegative and the inverse of $A_{n}$ is also nonnegative, the conditions $s^{n+1}, i^{n+1}, r^{n+1} \geq 0$ are satisfied.

If $c=d=0$ then the monotonicity of $s^{n}$ and $r^{n}$ can be deduced in the following way. From the first equation in (2.3) we obtain

$$
s^{n+1}=\frac{1-\tau(1-\sigma) p^{n}}{1+\tau \sigma p^{n}} s^{n}=\left(1-\frac{\tau p^{n}}{1+\tau \sigma p^{n}}\right) s^{n} .
$$

Then it follows from condition (C2) that the coefficient vector of $s^{n}$ is nonnegative and is not greater than one. Thus by the nonnegativity of $s^{n+1}$, we have $s^{n+1} \leq s^{n}$. The relation $r^{n+1} \geq r^{n}$ follows from the third equation in (2.3) in view of the nonnegativity of the vectors $i^{n}$ and $i^{n+1}$. This completes the proof.

The direct consequence of the previous lemmas is the following theorem that guarantees the validity of the qualitative properties [P1]-[P3].

Theorem 2.3. If conditions (C1)-(C2) are valid for all time-levels then properties [P1]-[P3] are satisfied.

We will see that conditions $(\mathrm{C} 1)-(\mathrm{C} 2)$ can be guaranteed a priori for all time levels by choosing the time step to be sufficiently small.

Now we consider some special forms of the model (2.1). A particular choice could be $\sigma=0, p^{n}=a i^{n}(a>0)$ but in this case the development of the disease on each subinterval would be independent of the disease states of the surrounding subintervals. The disease would develop on each interval separately, which is not interesting from practical point of view.

In the sequel, the dynamics of the development of the disease is defined by the vector

$$
p^{n}=\vartheta i^{n}+\frac{\varphi}{h^{2}} Q i^{n}
$$

where $\vartheta$ and $\varphi$ are positive parameters and $Q \in \mathbb{R}^{N \times N}$ has negative diagonal and nonnegative offdiagonal elements (that is $-Q$ is a so-called Z-matrix). We use the notations: $D=\operatorname{diag}(Q)$ (the diagonal part of $Q$ ) and $F=Q-D$ (the offdiagonal part). Thus, under our assumptions, $D \leq 0$ and $F \geq 0$. Moreover, let $D_{\max }=\|D\|_{\infty}$ and $F_{\max }=\|F\|_{\infty}$. (Clearly, $D_{\text {max }}=\max _{i=1, \ldots, N}\left\{-Q_{i i}\right\}$ and $\left.F_{\max }=\max _{i=1, \ldots, N}\left\{\sum_{j=1, j \neq i}^{N} Q_{i j}\right\}\right)$. We suppose that $F_{\max } \neq 0$, otherwise, the diseases on the different subintervals would be independent.

With the above defined vector $p^{n}$, we give two (an explicit and an implicit) special models with spatial dependence and investigate the conditions of the qualitative properties [P1]-[P3].

In the first model we set $\sigma=0$. Thus, (2.3) takes the form

$$
\begin{aligned}
& s^{n+1}=\left(1-\tau p^{n}\right) s^{n}+\tau d i^{n}+\tau(c+d) r^{n}, \\
& i^{n+1}=\tau s^{n} p^{n}+(1-\tau(b+d)) i^{n}, \\
& r^{n+1}=\tau b i^{n}+(1-\tau(c+d)) r^{n} .
\end{aligned}
$$

The qualitative properties of the model can be guaranteed by the next theorem.

Theorem 2.4. Consider the explicit discrete one-step iteration (2.6) with $p^{n}$ given in (2.5). Suppose that at the initial state $p^{0} \geq 0$, moreover assume that

$$
\tau \leq \min \left\{\tau_{1}, \tau_{2}\right\}
$$


where

$$
\tau_{1}=\frac{1}{\max \{c, b\}+d+D_{\max } \varphi M / h^{2}}, \quad \tau_{2}=\frac{1}{M\left(\vartheta+F_{\max } \varphi / h^{2}\right)}
$$

and $M=\max \left(s^{n}+i^{n}+r^{n}\right)$. Then the iteration satisfies the qualitative properties [P1]-[P3].

Proof. We apply Theorem 2.3 and show that if $s^{0}, i^{0}, r^{0} \geq 0$ then conditions (C1)-(C2) are true for all time levels. From the assumption $\tau \leq \tau_{1}$ in (2.7), the validity of (C1) follows immediately. Moreover, we have

$$
\tau p^{0}=\tau\left(\vartheta i^{0}+\frac{\varphi}{h^{2}} Q i^{0}\right) \leq \tau\left(\vartheta i^{0}+\frac{\varphi}{h^{2}} F i^{0}\right) \leq \tau M\left(\vartheta+\frac{F_{\max } \varphi}{h^{2}}\right) \leq 1
$$

and together with the condition $p^{0} \geq 0$ this yields that condition (C2) is satisfied for $n=0$. It follows from Lemma 2.2 that $s^{1} \geq 0, i^{1} \geq 0, r^{1} \geq 0$, that is $0 \leq i^{1} \leq s^{1}+i^{1}+r^{1} \leq M$, and the estimate (2.8) can be repeated changing the superscript 0 to 1 . Thus condition $\tau p^{1} \leq 1$ has been shown. Now we show that $p^{1} \geq 0$. Using the second equation in (2.6) and then (2.5), we can rewrite $p^{1}$ as

$$
\begin{aligned}
p^{1} & =\vartheta i^{1}+\frac{\varphi}{h^{2}} Q i^{1} \\
& =\vartheta\left(\tau s^{0} p^{0}+(1-(b+d) \tau) i^{0}\right)+\frac{\varphi}{h^{2}} Q\left(\tau s^{0} p^{0}+(1-(b+d) \tau) i^{0}\right) \\
& =(1-(b+d) \tau) \underbrace{\left(\vartheta i^{0}+\frac{\varphi}{h^{2}} Q i^{0}\right)}_{p^{0}}+\tau \vartheta s^{0} p^{0}+\tau \frac{\varphi}{h^{2}} Q\left(s^{0} p^{0}\right) \\
& =\left(1-(b+d) \tau+\tau \vartheta s^{0}\right) p^{0}+\tau \frac{\varphi}{h^{2}} Q\left(s^{0} p^{0}\right) .
\end{aligned}
$$

Due to the nonnegativity of the vector $s^{0} p^{0}$ and since $F \geq 0$, we have $Q\left(s^{0} p^{0}\right)=$ $(D+F)\left(s^{0} p^{0}\right) \geq D\left(s^{0} p^{0}\right) \geq-D_{\max } s^{0} p^{0}$. Hence, based on (2.9),

$$
p^{1} \geq\left(1-(b+d) \tau+\tau \vartheta s^{0}-\tau \frac{\varphi}{h^{2}} D_{\max } s^{0}\right) p^{0}=\left(1-\tau\left(b+d-\vartheta s^{0}+\frac{\varphi}{h^{2}} D_{\max } s^{0}\right)\right) p^{0} .
$$

The nonnegativity of $p^{1}$ can be guaranteed by the condition

$$
\tau\left(b+d-\vartheta s^{0}+\frac{\varphi}{h^{2}} D_{\max } s^{0}\right) \leq 1,
$$

which follows from the assumption $\tau \leq \tau_{1}$ in (2.7). The above expressions show that condition (C2) is also valid with $n=1$.

Then we can apply induction for $n$. The step from $n$ to $n+1$ can be carried out similarly as we did from $n=0$ to $n=1$. This completes the proof.

Thus if $s^{0} \geq 0, i^{0} \geq 0, r^{0} \geq 0$ and the vector $p^{0}$ defined with $i^{0}$ is also nonnegative then with a suitably chosen (sufficiently small) time step the required qualitative properties are fulfilled for the explicit model.

Let us turn to the second model. With the choice $\sigma=1$, system (2.3) takes the form

$$
\left[\begin{array}{ccc}
1+\tau p^{n} & -\tau d & -\tau(c+d) \\
-\tau p^{n} & 1+\tau(b+d) & 0 \\
0 & -\tau b & 1+\tau(c+d)
\end{array}\right]\left[\begin{array}{c}
s^{n+1} \\
i^{n+1} \\
r^{n+1}
\end{array}\right]=\left[\begin{array}{c}
s^{n} \\
i^{n} \\
r^{n}
\end{array}\right]
$$

The qualitative properties [P1]-[P3] can be guaranteed for the above model by the following theorem. 
Theorem 2.5. Consider the implicit discrete one-step iteration (2.10) with $p^{n}$ given in (2.5). Suppose that at the initial state $p^{0} \geq 0$ and

$$
\tau \leq \begin{cases}\left(\left(D_{\max } \varphi / h^{2}-\vartheta\right) M\right)^{-1}, & \text { if } h<h^{\star}, \\ \text { arbitrary, } & \text { if } h \geq h^{\star},\end{cases}
$$

where $h^{\star}=\left(D_{\max } \varphi / \vartheta\right)^{1 / 2}$. Then the iteration satisfies the qualitative properties [P1]-[P3].

Proof. We apply Theorem 2.3. We show that if $s^{0}, i^{0}, r^{0} \geq 0$ then conditions (C1)-(C2) are valid for all time steps. Let us notice, that with $\sigma=1$ conditions (C1)-(C2) simplify only to $p^{n} \geq 0$.

Based on the assumptions of the theorem we have $p^{0} \geq 0$. Lemma 2.2 implies that $s^{1} \geq 0$, $i^{1} \geq 0, r^{1} \geq 0$, moreover $i^{1} \leq M$. We show that $p^{1} \geq 0$. Using the iteration (2.10) and then (2.5), we obtain

$$
\begin{aligned}
p^{1}= & \vartheta i^{1}+\frac{\varphi}{h^{2}} Q i^{1} \\
= & \vartheta\left(\frac{\tau}{1+\tau(b+d)} s^{1} p^{0}+\frac{1}{1+\tau(b+d)} i^{0}\right) \\
& +\frac{\varphi}{h^{2}} Q\left(\frac{\tau}{1+\tau(b+d)} s^{1} p^{0}+\frac{1}{1+\tau(b+d)} i^{0}\right) \\
= & \frac{1}{1+\tau(b+d)} \underbrace{\left(\vartheta i^{0}+\frac{\varphi}{h^{2}} Q i^{0}\right)}_{p^{0}}+\frac{\tau}{1+\tau(b+d)}\left(\vartheta s^{1} p^{0}+\frac{\varphi}{h^{2}} Q\left(s^{1} p^{0}\right)\right) \\
= & \frac{1}{1+\tau(b+d)} p^{0}+\frac{\tau}{1+\tau(b+d)}\left(\vartheta s^{1} p^{0}+\frac{\varphi}{h^{2}} Q\left(s^{1} p^{0}\right)\right) .
\end{aligned}
$$

Due to the nonnegativity of the vector $s^{1} p^{0}$ we have $Q\left(s^{1} p^{0}\right)=(D+F)\left(s^{1} p^{0}\right) \geq D\left(s^{1} p^{0}\right) \geq$ $-D_{\max } s^{1} p^{0}$. Hence, based on (2.12),

$$
p^{1} \geq \frac{1}{1+\tau(b+d)}\left(1+\tau\left(\vartheta-\frac{\varphi D_{\max }}{h^{2}}\right) s^{1}\right) p^{0} .
$$

If $h \geq h^{\star}$ then the right-hand side is nonnegative. In the case of $h<h^{\star}$ the nonnegativity is guaranteed by the condition $\tau \leq\left(\left(D_{\max } \varphi / h^{2}-\vartheta\right) M\right)^{-1}$. Thus, condition (2.11) guarantees the nonnegativity of $p^{1}$. Then we apply induction for $n$. The step from $n$ to $n+1$ can be showed as we did in the step from 0 to 1 . This completes the proof.

\section{Discretizations of continuous models and their qualitative prop- erties}

In this section we show how the system (1.3) in the one-dimensional case can be discretized in the form discussed in the previous section and we give sufficient conditions for the model parameters and the discretization mesh that guarantee the qualitative properties [P1]-[P3]. In the remainder of the paper, we use the special choice $N(x)=[x-\delta, x+\delta]$ with some positive parameter $\delta>0$.

In order to discretize in space and in time, we define a uniform spatial grid

$$
\omega_{h}=\left\{x_{k} \in[0, l] \mid x_{k}=-h / 2+k h, k=1, \ldots, N, h=l / N\right\}
$$


and a time step $\tau>0$. The functions $S, I$ and $R$ are approximated respectively by the vectors $s^{n}, i^{n}$ and $r^{n}$ at the $n$th time level $t=n \tau$. For example, the value $s_{k}^{n}$, the $k$-th element of the vector $s^{n}$, approximates the value $S\left(x_{k}, n \tau\right)$. For $n=0$, the grid functions are known from the initial conditions.

We discuss two types of discretization methods: numerical integration and Taylor expansion with finite difference discretization.

Method of numerical integration. We set $h=\delta$ and approximate the integral (1.2) in (1.3) by the trapezoidal rule. We apply the rule separately on the intervals $[x-h, x]$ and $[x, x+h]$ and add the results to get a suitable approximation of the integral. To help this, we extend the grid $\omega_{h}$ with the virtual points $x_{0}=x_{1}-h$ and $x_{N+1}=x_{N}+h$. At the point $x=x_{k}(k=1, \ldots, N)$ and at the $n$th time level we get the approximation

$$
\int_{N\left(x_{k}\right)} W\left(\left|x^{\prime}-x_{k}\right|\right) I\left(x^{\prime}, n \tau\right) \mathrm{d} x^{\prime} \approx \frac{h}{2}\left(W(\delta) i_{k-1}^{n}+W(\delta) i_{k+1}^{n}+2 W(0) i_{k}^{n}\right)=p_{k}^{n} .
$$

This value will be denoted by $p_{k}^{n}$, and the vector $p^{n}$ has the form (2.5) with

$$
\vartheta=h(W(0)+W(\delta)), \quad \varphi=\frac{h^{3}}{2} W(\delta)
$$

and

$$
Q=\left[\begin{array}{ccccccc}
-1 & 1 & & & & & \\
1 & -2 & 1 & & & & \\
& 1 & -2 & 1 & & & \\
& & \ddots & \ddots & \ddots & & \\
& & & & 1 & -2 & 1 \\
& & & & & 1 & -1
\end{array}\right]
$$

Here homogeneous Neumann boundary conditions were applied, thus we set $i_{0}^{n}=i_{1}^{n}$ and $i_{N+1}^{n}=i_{N}^{n}$. The fully discretized problem takes the final form (2.1) with the above notations. For the matrix $Q$ we have $F_{\max }=D_{\max }=2$.

Remark 3.1. The choice $h=\delta$ is motivated by the following considerations. When we choose $h<\delta$ then the numerical integration formulas become much more complicated. At the same time, by the choice $h>\delta$, the numerical integration formulas on $N\left(x_{k}\right)$ would use only the value $i_{k}^{n}$. This would mean that the disease on each subinterval would develop independently of the surrounding subintervals, as we noted in the previous section.

We get the following sufficient conditions of the qualitative properties [P1]-[P3].

Theorem 3.2. Let $\delta>0$ be given. If in the discretization (2.1) of system (1.3) we choose $h=\delta$, $\sigma=0$ and the composite trapezoidal rule, then we arrive at system (2.6) with $p^{n}$ defined in (2.5) and parameters $\vartheta, \varphi$ and $Q$ given in (3.1) and (3.2), respectively. If at the initial state $p^{0} \geq 0$, moreover

$$
\tau \leq \min \left\{\frac{1}{\max \{c, b\}+d+h M W(\delta)}, \frac{1}{M h(W(0)+2 W(\delta))}\right\}
$$

then the discrete scheme satisfies the qualitative properties [P1]-[P3].

Proof. The statement follows from Theorem 2.4.

In the implicit case we do not get upper bound for the time step $\tau$. 
Theorem 3.3. Let $\delta>0$ be given. If in the discretization of system (1.3) we choose $h=\delta, \sigma=1$ and the composite trapezoidal rule, then we arrive at system (2.10) with $p^{n}$ defined in (2.5) and parameters $\vartheta, \varphi$ and $Q$ given in (3.1) and (3.2), respectively. If at the initial state $p^{0} \geq 0$ then the discrete scheme satisfies the qualitative properties [P1]-[P3].

Proof. We apply Theorem 2.5. In view of the relation

$$
\vartheta-\frac{\varphi D_{\max }}{h^{2}}=h(W(0)+W(\delta))-\frac{2 h^{3} W(\delta) / 2}{h^{2}}=h W(0) \geq 0,
$$

the condition $h \geq h^{\star}$ is satisfied, thus the time step can be chosen to be arbitrary. This completes the proof.

Taylor expansion and finite difference approximation. Let us approximate the function $I$ with its second order Taylor polynomial in $x$. In this way we arrive at the system [7]

$$
\begin{aligned}
S_{t}^{\prime} & =-S\left(\vartheta I+\varphi I_{x x}^{\prime \prime}\right)+d I+(c+d) R, \\
I_{t}^{\prime} & =S\left(\vartheta I+\varphi I_{x x}^{\prime \prime}\right)-(b+d) I, \\
R_{t}^{\prime} & =b I-(c+d) R
\end{aligned}
$$

where

$$
\vartheta=\int_{-\delta}^{\delta} W(|u|) \mathrm{d} u, \quad \varphi=\frac{1}{2} \int_{-\delta}^{\delta} u^{2} W(|u|) \mathrm{d} u
$$

We notice that the values in (3.1) are the approximations of the above integrals with the trapezoidal rule.

We solve the system (3.4) numerically by the finite difference method on the finite spatial interval $[0, l]$. At the two ends of the interval homogeneous Neumann boundary conditions are applied.

Let us consider the following explicit discretization scheme, where the time differences and the second order spatial derivatives are approximated in the standard way with forward and centred differences, respectively:

$$
\begin{aligned}
& \frac{s_{k}^{n+1}-s_{k}^{n}}{\tau}=-s_{k}^{n}\left(\vartheta i_{k}^{n}+\varphi \frac{i_{k-1}^{n}-2 i_{k}^{n}+i_{k+1}^{n}}{h^{2}}\right)+d i_{k}^{n}+(c+d) r_{k}^{n}, \\
& \frac{i_{k}^{n+1}-i_{k}^{n}}{\tau}=s_{k}^{n}\left(\vartheta i_{k}^{n}+\varphi \frac{i_{k-1}^{n}-2 i_{k}^{n}+i_{k+1}^{n}}{h^{2}}\right)-(b+d) i_{k}^{n}, \\
& \frac{r_{k}^{n+1}-r_{k}^{n}}{\tau}=b i_{k}^{n}-(c+d) r_{k}^{n},
\end{aligned}
$$

$k=1, \ldots, N$. From the homogeneous Neumann boundary conditions, it follows that $i_{0}^{n}=i_{1}^{n}$ and $i_{N+1}^{n}=i_{N}^{n}$ for all $n=1,2, \ldots$ The above scheme can be written in the one-step vector iteration form (2.6) with $p^{n}$ defined in (2.5), and with $Q$ defined in (3.2). Theorem 2.4 implies the following statement directly.

Theorem 3.4. Let us suppose that at the initial state $p^{0} \geq 0$, moreover assume that

$$
\tau \leq \min \left\{\frac{1}{\max \{c, b\}+d+2 \varphi M / h^{2}}, \frac{1}{M\left(\vartheta+2 \varphi / h^{2}\right)}\right\} .
$$

Then the explicit finite difference discretization (3.6) of (3.4) satisfies the qualitative properties [P1][P3]. 
After the investigation of the explicit scheme for the system (3.4), we turn to the implicit scheme

$$
\begin{aligned}
& \frac{s_{k}^{n+1}-s_{k}^{n}}{\tau}=-s_{k}^{n+1}\left(\vartheta i_{k}^{n}+\varphi \frac{i_{k-1}^{n}-2 i_{k}^{n}+i_{k+1}^{n}}{h^{2}}\right)+d i_{k}^{n+1}+(c+d) r_{k}^{n+1}, \\
& \frac{i_{k}^{n+1}-i_{k}^{n}}{\tau}=s_{k}^{n+1}\left(\vartheta i_{k}^{n}+\varphi \frac{i_{k-1}^{n}-2 i_{k}^{n}+i_{k+1}^{n}}{h^{2}}\right)-(b+d) i_{k}^{n+1}, \\
& \frac{r_{k}^{n+1}-r_{k}^{n}}{\tau}=b i_{k}^{n+1}-(c+d) r_{k}^{n+1} .
\end{aligned}
$$

Since (3.8) is the model (2.10) with the matrix $Q$ in (3.2), similarly to the explicit case, Theorem 2.5 can be applied. We get the following result.

Theorem 3.5. Let us suppose that at the initial state $p^{0} \geq 0$ and

$$
\tau \leq \begin{cases}\left(\left(2 \varphi / h^{2}-\vartheta\right) M\right)^{-1}, & \text { if } h<h^{\star}, \\ \text { arbitrary, } & \text { if } h \geq h^{\star},\end{cases}
$$

where $h^{\star}=(2 \varphi / \vartheta)^{1 / 2}$. Then the implicit finite difference discretization (3.8) of (3.4) with $p^{n}$ given in (2.5) satisfies the qualitative properties [P1]-[P3].

Comparing conditions (3.7) and (3.9) one can easily see that the condition for the time step $\tau$ is less restrictive in the case of the implicit scheme. Thus from the qualitative point of view the implicit scheme can be preferred.

\section{Numerical tests}

We show some numerical tests that demonstrate the results given in the previous section. For the sake of brevity, we consider only the implicit model. As we have seen in the previous section, the restrictions for the mesh parameters are less severe than in the explicit case, although, in the implicit case we have to solve a system of linear equations in each iteration step. We solve the system with the standard Gauss elimination process.

First we consider tests that demonstrate the result of Theorem 3.5. In these simulations we use the weighting function $W(u)=1-|u| / \delta$ with $|u| \in[0, \delta]$. With the above choice we will have $\vartheta=\delta$ and $\varphi=\delta^{3} / 12$. We set $l=10, \delta=0.07, b=0.05, c=0.03$ or $c=0$ and $d=0.01$ or $d=0$. The spatial step size is set to $h=0.025$. The initial density functions for the investigated three subpopulations can be seen on the left panel of Figure 4.1. The right panel shows the total population density function. Obviously, for this setting the condition $p^{0} \geq 0$ is satisfied. The figure shows a population with a constant basic susceptible density 1.6, excepting the neighbourhood of the point $x=2$, where the density of the susceptibles is higher (around 2.3). Infected individuals are located only around the point $x=5$. Their maximal density is 1.2 . Here the $43 \%$ of the individuals are infected in the initial time instant. For these initial functions the maximum of the sum of the density functions is $M=2.8$.

When we solve the system (3.4) with the parameters defined above using the implicit finite difference discretization (3.8) then in order to guarantee the properties [P1]-[P3] we have to choose the time-step according to the relation (3.9). In view that $h^{\star}=0.02858$ and $h=0.025$, the properties can be guaranteed by choosing the time step to be $\tau \leq 16.6371$. We notice that in the explicit case we would have to choose much smaller time steps, namely below 1.4120 .

When we choose $\tau=50$ (thus above the given bound), $c=0.03$ and $d=0.01$ then we get the qualitatively inadequate solution given in Figure 4.2. Namely, the number of the infectives 

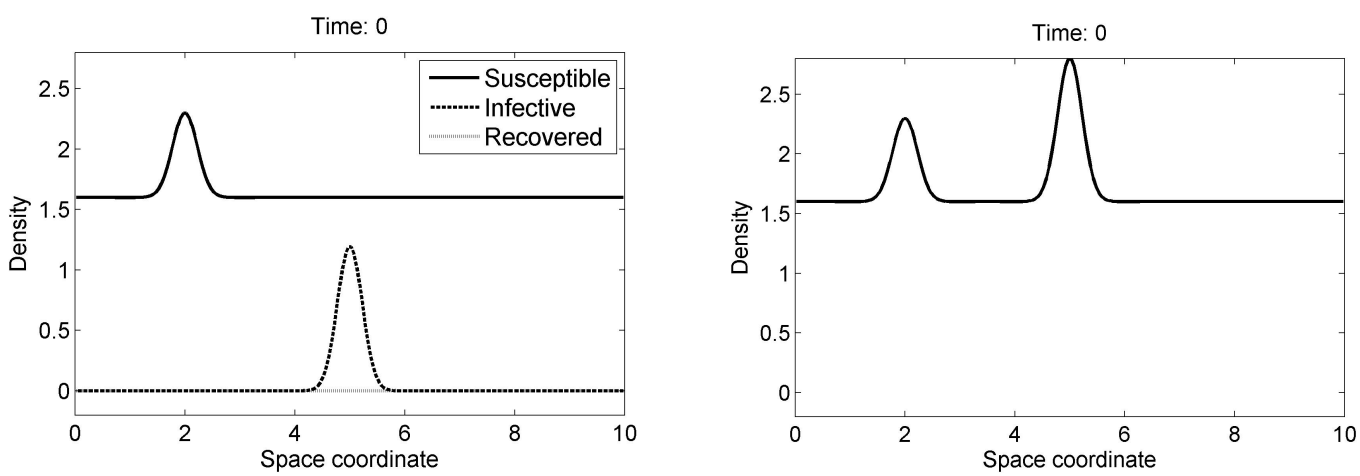

Figure 4.1: The initial density functions and the population density function.

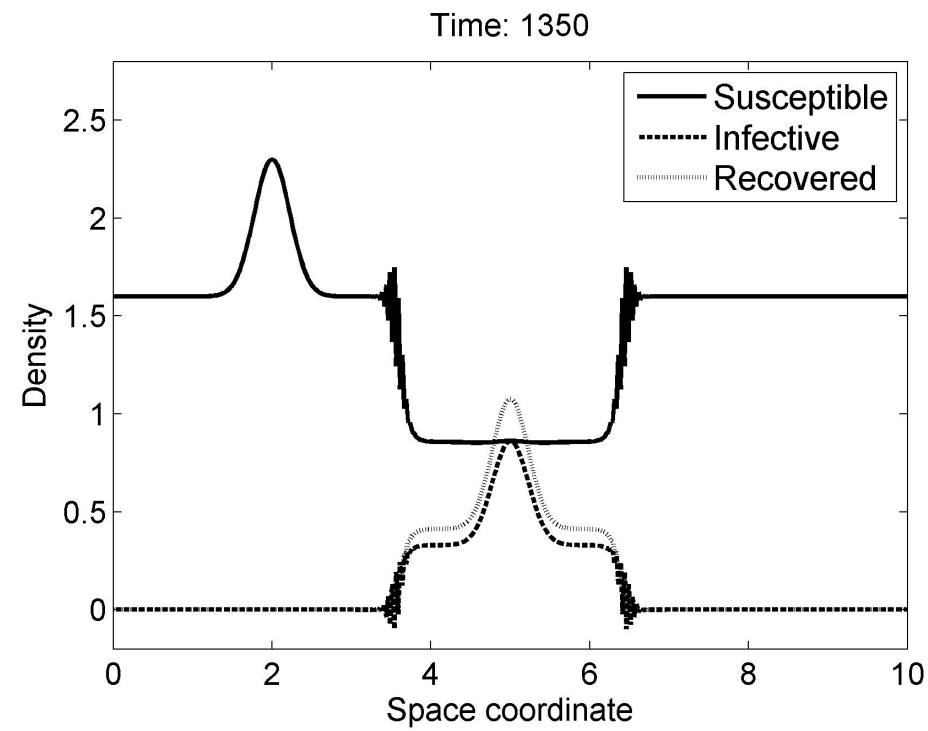

Figure 4.2: A qualitatively inadequate solution with the implicit scheme (3.8) and with $\tau=50$ at the time level $t=1350$. The number of infectives is negative around the points $x=3.5$ and $x=6.5$.

is negative around the points $x=3.5$ and $x=6.5$. During the iteration process we get negative concentration values several times but at the same time the sum $s_{k}^{n}+i_{k}^{n}+r_{k}^{n}$ remains constant at fixed points $x_{k}$. After these iterations, the stationary solution will be qualitatively correct (Figure 4.3). We notice that $S$ is not a constant function. Because $\varphi$ is relatively small in this example the change of $S$ is not noticeable in the figure.

When we choose $\tau=15$, thus below the computed upper bound, then the solution will be qualitatively correct (Figure 4.4). In the models epidemic waves can be seen. These waves occur because the concentration of the susceptibles are high enough to conduct such waves. In Figure 4.4 the epidemic wave reaches the point $x=2$, where the number of susceptibles drop down drastically.

For the case when $c=d=0$ similar phenomena can be seen. With time steps below the computed upper bound the solution is qualitatively correct, but above the bound incorrect solutions can occur. Here after a certain period of time the disease dies out and leaves back only recovered individuals and some susceptibles who were not infected by the disease. This final state, obtained by the implicit method (3.8) with $\tau=16$ can be seen in Figure 4.5. 


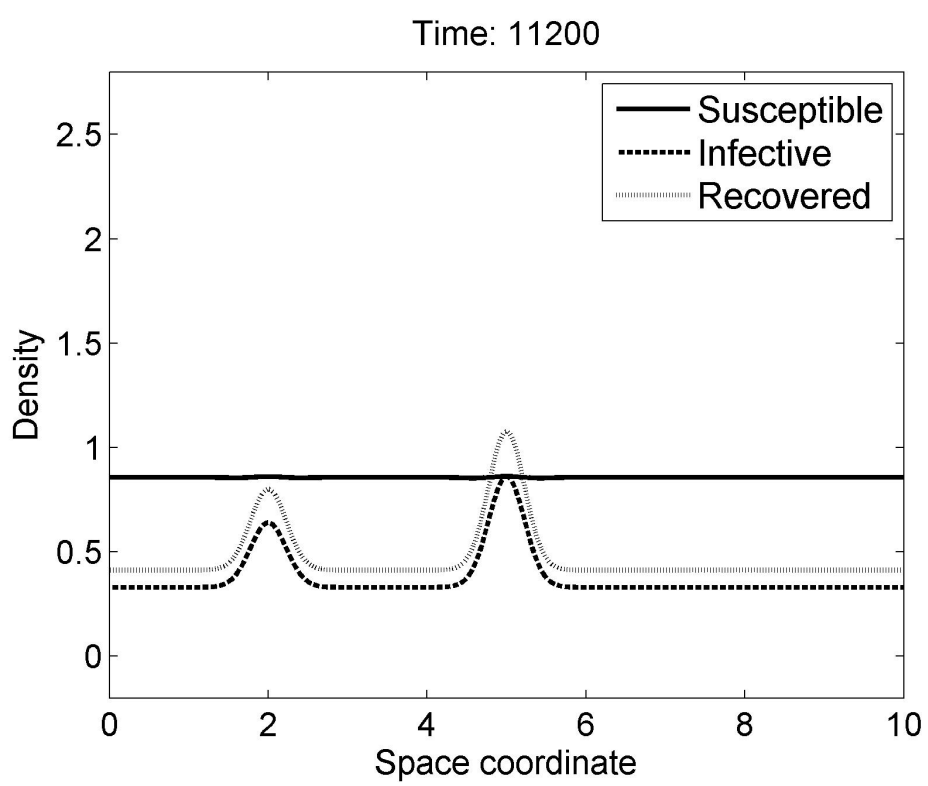

Figure 4.3: The stationary state at the time level $t=11200$ using implicit scheme (3.8) and $\tau=50$.

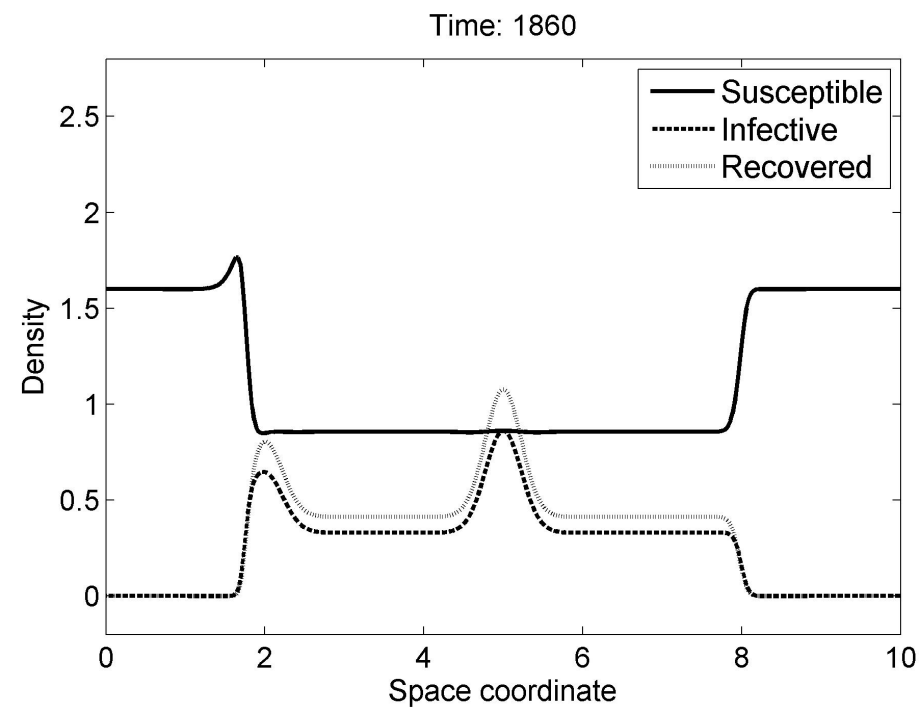

Figure 4.4: Solution with the implicit scheme (3.8) and $\tau=15$ at the time level $t=1860$.

Now we demonstrate Theorem 3.3. We set the weighting function to $W(u)=(-4|u| / \delta+$ $5) / 6,|u| \in[0, \delta]$ and obtain the parameters $\vartheta=h, \varphi=h^{3} / 12$. The parameters are set to be $l=10, \delta=0.05, b=0.05, c=0.03, d=0.01$. The spatial step size is set to $h=0.05=\delta$. The initial functions are the same as in the previous tests (Figure 4.1). Based on the statement of Theorem 3.3, the numerical solution will be qualitatively adequate (satisfies properties [P1][P3]) independently of the time step. Indeed, when we choose $\tau=200$, the iteration produces qualitatively correct states. The state at $t=26400$ and the stationary state at $t=70000$ can be seen in Figure 4.6.

Thus the numerical tests verify the results obtained in the previous section. 
Time: 9000

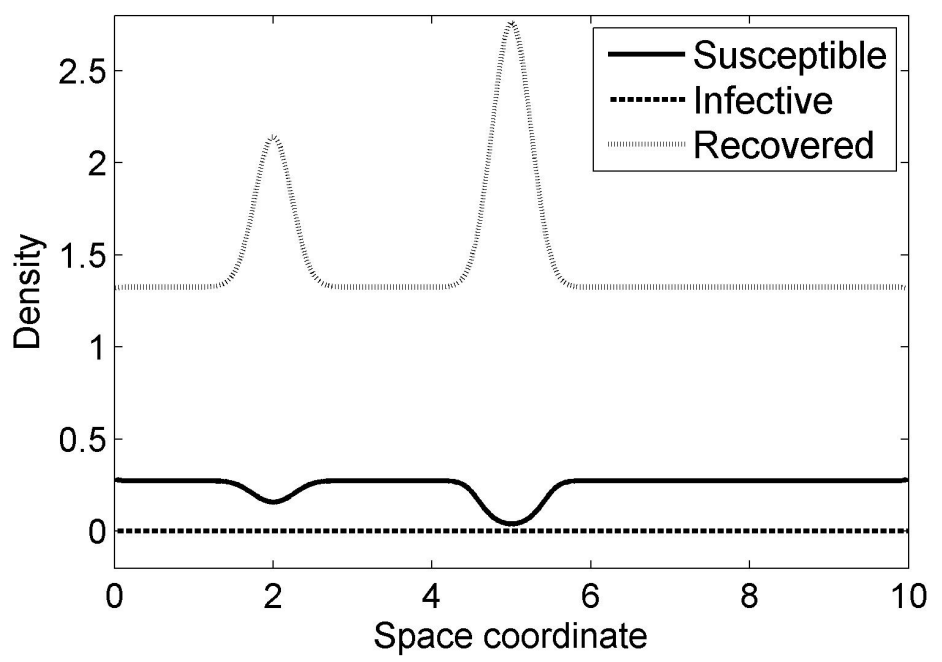

Figure 4.5: The final state at the time level $t=9000$. The parameters are: $c=d=0, \tau=15$ and the implicit scheme (3.8) is used.
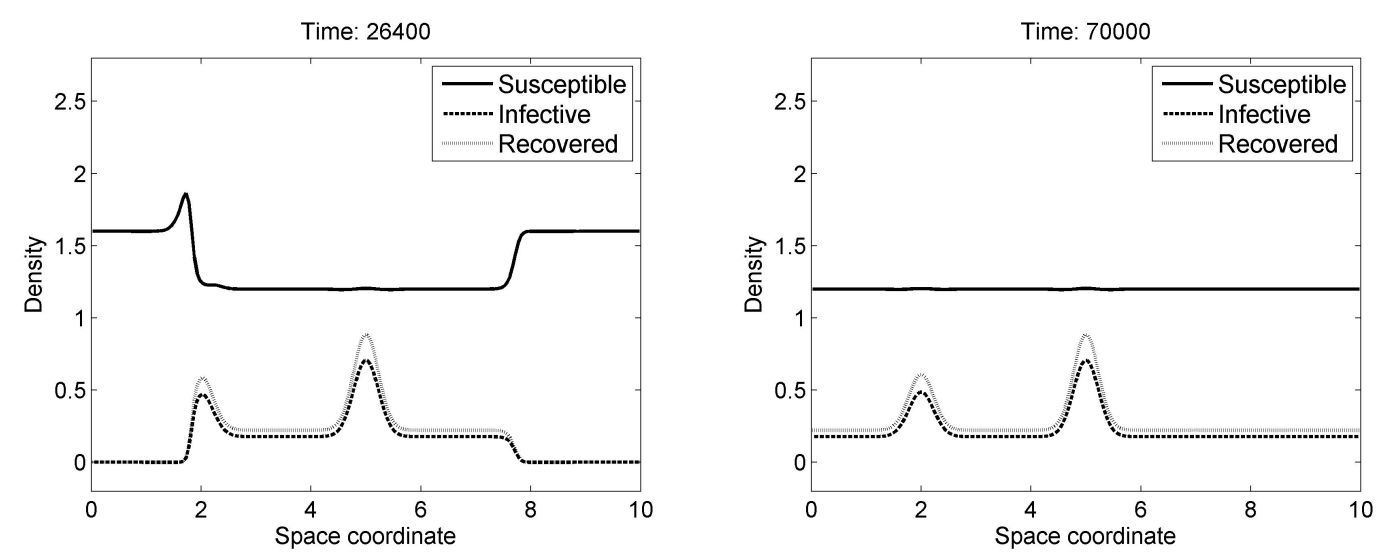

Figure 4.6: The state at $t=26400$ and the final state at $t=70000$ obtained with the time step $\tau=200$.

\section{Summary and future work}

We defined a discrete one-step iteration that can be considered as some suitable discretization of a continuous disease propagation model that takes into account the spacial inhomogeneity. We gave sufficient conditions that guarantee the basic characteristic properties of the disease propagation to the discrete iteration model and deduced sufficient conditions for the choice of the mesh parameters in the discretization processes. We considered two discretization methods: one with numerical integration and one with Taylor expansion in combination with finite differences. Using our results, we can guarantee a priori a qualitatively adequate numerical solution for the continuous model by choosing the meshes appropriately.

We are planning to extend our investigation for higher dimensional problems. It is also interesting to consider other important qualitative properties, other discretization methods or other boundary conditions. A larger step would be the inclusion of the motion of the individuals into the model and repeat a similar qualitative investigation. 


\section{Acknowledgements}

The authors gratefully acknowledge the constructive comments offered by the anonymous referee. This research was supported by the Hungarian Scientific Research Fund under the grant OTKA 112157.

\section{References}

[1] R. M. Anderson, Population dynamics of infectious diseases: theory and applications, Chapman and Hall, London-New York, 1982

[2] A. Berman, R. J. Plemmons, Nonnegative matrices in the mathematical sciences, Academic Press, New York, 1979. MR0544666

[3] F. Brauer, C. Castillo-Chávez, Mathematical models in population biology and epidemiology, Springer, New York, 2001. MR1822695

[4] V. Capasso, Mathematical structures of epidemic systems, Lecture Notes in Biomathematics, Vol. 97, Springer, 2008. MR2722340

[5] S. Chinviriyasit, W. Chinviriyasit, Numerical modelling of an SIR epidemic model with diffusion, Appl. Math. Comp. 216(2010), 395-409. MR2601507

[6] I. FARAgó, R. Horváth, On some qualitatively adequate discrete space-time models of epidemic propagation, J. Comput. Appl. Math. 293(2016), 45-54. MR3394200; url

[7] D. S. Jones, B. D. Sleeman, Differential equations and mathematical biology, Chapman \& Hall/CRC Mathematical Biology and Medicine Series, CRC Press, 2011. MR1967145

[8] J. P. Keller, L. Gerardo-Giorda, A. Veneziani, Numerical simulation of a susceptibleexposed-infectious space-continuous model for the spread of rabies in raccoons across a realistic landscape, J. Biol. Dyn. 7(2013), suppl. 1, 31-46. MR3196365; url

[9] D. G. Kendall, Mathematical models of the spread of infection, in: Mathematics and computer science in biology and medicine, H.M.S.O., London, 1965, 213-225.

[10] W. O. Kermack, A. G. McKendrick, A contribution to the mathematical theory of epidemics, Proc. R. Soc. Lond. Ser. A Math. Phys. Eng. Sci. 115(1927), No. 772, 700-721. url

[11] M. Kolev, M. Koleva, L. Vulkov, Two positivity preserving flux limited, second-order numerical methods for a haptotaxis model, Numer. Methods Partial Differential Equations 29(2013), No. 4, 1121-1148. MR3053860 